\title{
Angular dependence of Josephson currents in unconventional superconducting junctions
}

\author{
T. Yokoyama, ${ }^{1}$ Y. Sawa, ${ }^{1}$ Y. Tanaka, ${ }^{1}$ and A. A. Golubov ${ }^{2}$ \\ ${ }^{1}$ Department of Applied Physics, Nagoya University, Nagoya, 464-8603, Japan \\ and CREST, Japan Science and Technology Corporation (JST) Nagoya, 464-8603, Japan \\ ${ }^{2}$ Faculty of Science and Technology, University of Twente, 7500 AE, Enschede, The Netherlands
}

(Received 26 September 2006; published 3 January 2007)

\begin{abstract}
Josephson effect in junctions between unconventional superconductors is studied theoretically within the model describing the effects of interface roughness. The particularly important issue of applicability of the frequently used Sigrist-Rice (SR) formula for Josephson current in $d$-wave superconductor/insulator/ $d$-wave superconductor junctions is addressed. We show that although the SR formula is not applicable in the ballistic case, it works well for rough interfaces when the diffusive normal metal regions exist between the $d$-wave superconductor and the insulator. It is shown that the SR approach only takes into account the component of the $d$-wave pair potential symmetric with respect to an inversion around the plane perpendicular to the interface. Similar formula can be derived for general unconventional superconductors with arbitrary angular momentum $l$.
\end{abstract}

DOI: 10.1103/PhysRevB.75.020502

PACS number(s): 74.20.Rp, 74.50.+r, 74.70.Kn

A number of phase-sensitive experiments have convincingly demonstrated the realization of $d$-wave pairing state in high- $T_{C}$ cuprates. ${ }^{1-4}$ Because of such unconventional symmetry, the study of Josephson effect in high- $T_{C}$ superconducting (HTS) junctions attracted a lot of interest. A while ago, a simple formula for the Josephson current of $d$-wave superconductor/insulator/ $d$-wave superconductor (DID) junctions was proposed by Sigrist and Rice (SR)..$^{5}$ According to the SR formula, the Josephson current is proportional to $\cos 2 \alpha \cos 2 \beta$, where the $\alpha(\beta)$ denotes the angle between the normal to the interface and the crystal axis of the left(right) $d$-wave superconductor. ${ }^{5}$

Although the SR formula can explain experiments with the so-called $\pi$ junctions, ${ }^{1,2}$ this formula does not take into account the effect of midgap Andreev resonant states (MARS) formed at junction interfaces. ${ }^{6,7}$ Actually, as shown in Ref. 8, SR formula does not work in ballistic $d$-wave junctions for $\alpha \neq 0$ and $\beta \neq 0$ where MARS influence severely the charge transport at low temperatures. It was shown both theoretically ${ }^{9,10}$ and experimentally ${ }^{11,12}$ that MARS induce a nonmonotonic temperature dependence of the maximum Josephson current in DID junctions. On the other hand, SR formula has been extensively used to analyze experiments with various types of HTS Josephson junctions. ${ }^{13-15}$ Experiments with HTS junctions are of high importance for basic understanding of high- $T_{c}$ superconductivity since they may provide information on possible subdominant admixtures to the $d$-wave symmetry. ${ }^{16-18}$ Therefore it is of fundamental interest to understand the physical mechanisms that determine the angular dependence of Josephson current in HTS junctions. For this reason, the determination of the conditions of applicability of the SR formula is an important issue which is addressed in the present paper.

In the following, we study the Josephson current in $D / \mathrm{DN} / I / \mathrm{DN} / D$ junctions, where $D N$ denotes diffusive normal metal and could be formed between the insulator and $d$-wave superconductors. The calculations are based on the quasiclassical Green's function method applicable to unconventional superconductor junctions. ${ }^{19,20}$ We find that the re- sulting Josephson current in $D / \mathrm{DN} / I / \mathrm{DN} / D$ junctions is well fitted by the SR formula. Near the transition temperature, it is proven analytically that Josephson current follows the SR formula. We also confirm that this formula does not hold in the ballistic junctions. It is clarified that in the SR formula, the component of the pair potential that is antisymmetric by the inversion operation around the plane perpendicular to the interface is neglected. We also study $p$-wave superconductor/diffusive normal metal/insulator/diffusive normal metal $/ p$-wave superconductor $(P / \mathrm{DN} / I / \mathrm{DN} / P)$ junctions. The resulting Josephson current is also well fitted by $\cos \alpha \cos \beta$, where $\alpha(\beta)$ denotes the angle between the crystal axis of left (right) $p$-wave superconductor and the normal to the interface. This is a corresponding version of the SR formula in the $p$-wave superconductor junctions. Furthermore, it is possible to extend the theory for an unconventional superconductor (US) with arbitrary angular momentum $l$. For US/DN/I/DN/US junctions, the expected Josephson current is proportional to $\cos l \alpha \cos l \beta$. The obtained results may serve as a guide for the analysis of the experiments in unconventional superconductor junctions.

Before we proceed with a formal discussion, let us provide qualitative arguments on the physical meaning of the SR formula and explain why it holds in the diffusive junctions. First, we consider $d$-wave superconductor junctions. The pair potentials of left and right $d$-wave superconductors are, respectively, expressed by $\Delta_{L}$ $=\Delta\left[f_{S L}(\phi)+f_{A S L}(\phi)\right] \exp (-i \Psi)$, and $\Delta_{R}=\Delta\left[f_{S R}(\phi)+f_{A S R}(\phi)\right]$, with $f_{S L}(\phi)=\cos 2 \phi \cos 2 \alpha, f_{A S L}(\phi)=\sin 2 \phi \sin 2 \alpha, f_{S R}(\phi)$ $=\cos 2 \phi \cos 2 \beta, f_{A S R}(\phi)=\sin 2 \phi \sin 2 \beta$, where $\phi$ is the injection angle measured from the interface normal, $\Delta$ denotes the maximum value of the pair potential and $\Psi$ is the phase difference across the junction. The terms proportional to $\cos 2 \phi$, i.e., $f_{S L}(\phi)$ and $f_{S R}(\phi)$, correspond to the $d_{x^{2}-y^{2} \text {-wave }}$ pair potential and the terms proportional to $\sin (2 \phi)$, i.e., $f_{A S L}(\phi)$ and $f_{A S R}(\phi)$, correspond to the $d_{x y}$-wave pair potential, respectively. Here, $f_{S L}(\phi)=f_{S L}(-\phi), f_{S R}(\phi)=f_{S R}(-\phi)$, $f_{A S L}(\phi)=-f_{A S L}(-\phi)$, and $f_{A S R}(\phi)=-f_{A S R}(-\phi)$ are satisfied. In the actual calculation of Josephson current in 
(a)

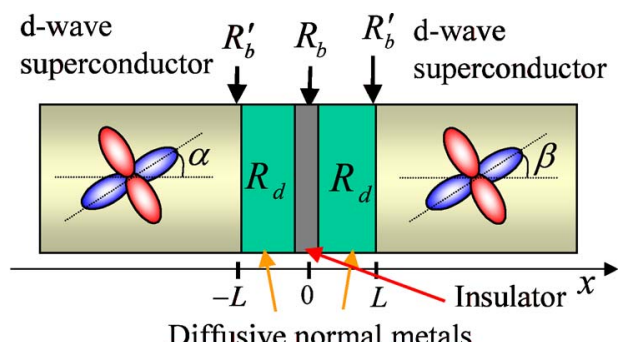

Diffusive normal metals

(b)

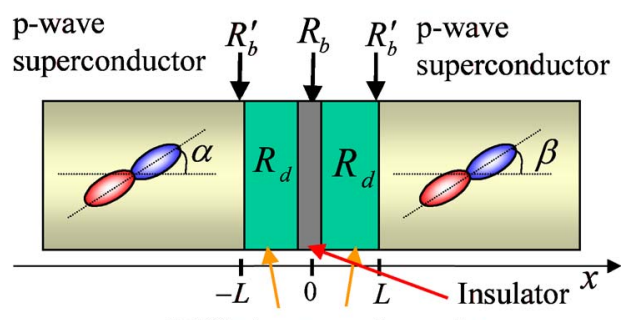

Diffusive normal metals

FIG. 1. (Color online) Schematic illustration of the models of (a) $D / D N / I / D N / D$ and (b) $P / D N / I / D N / P$ junctions.

$D / \mathrm{DN} / I / \mathrm{DN} / D$ junctions, we have to take an average over the various $\phi$. Due to the impurity scattering in DN, the average is taken for the left and right $D / D N$ interface independently. Then we can drop $f_{A S L}(\phi)$ and $f_{A S R}(\phi)$ and arrive at the SR formula, where only the terms $f_{S L}(\phi)$ and $f_{S R}(\phi)$ remain which do not change sign by exchanging $\phi$ for $-\phi$. This fact is in accordance with the recent result that the proximity effect is absent in the case of $d_{x y}$-wave pair potential. ${ }^{19}$ Consequently, the resulting Josephson current is proportional to $\cos 2 \alpha \cos 2 \beta$.

Similar arguments apply to $p$-wave junctions. In this case, $f_{S L}(\phi)=\cos \phi \cos \alpha, f_{A S L}(\phi)=\sin \phi \sin \alpha, f_{S R}(\phi)$ $=\cos \phi \cos \beta$, and $f_{A S R}(\phi)=\sin \phi \sin \beta$ are satisfied. The terms proportional to $\cos \phi$, i.e., $f_{S L}(\phi)$ and $f_{S R}(\phi)$, correspond to the $p_{x}$-wave pair potential and the terms proportional to $\sin \phi$, i.e., $f_{A S L}(\phi)$ and $f_{A S R}(\phi)$, correspond to the $p_{y}$-wave pair potential. In the actual calculation for $P / \mathrm{DN} / I / \mathrm{DN} / P$ junctions, functions $f_{A S L}(\phi)$ and $f_{A S R}(\phi)$ vanish after averaging over angle $\phi$. This is consistent with our previous results that the pair potential with $p_{y}$-wave symmetry does not contribute to the proximity effect. ${ }^{21,22}$

Next we formulate the junction model and basic equations starting from the $d$-wave case. We consider ballistic $D I D$ and $D / \mathrm{DN} / I / \mathrm{DN} / D$ junctions. The DN has a resistance $R_{d}$ and a length $L$ much larger than the mean free path. The DN/D interfaces located at $x= \pm L$ have the resistance $R_{b}^{\prime}$, while the $\mathrm{DN} / I$ interface at $x=0$ has the resistance $R_{b}$. We model infinitely narrow insulating barriers by the delta function $U(x)$ $=H^{\prime} \delta(x+L)+H \delta(x)+H^{\prime} \delta(x-L)$. The resulting transparencies of the junctions $T_{m}$ and $T_{m}^{\prime}$ are given by $T_{m}$ $=4 \cos ^{2} \phi /\left(4 \cos ^{2} \phi+Z^{2}\right)$ and $T_{m}^{\prime}=4 \cos ^{2} \phi /\left(4 \cos ^{2} \phi+Z^{\prime 2}\right)$, where $Z=2 H / v_{F}$ and $Z^{\prime}=2 H^{\prime} / v_{F}$ are dimensionless constants (we take $\hbar=k_{B}=1$ in this paper) and $v_{F}$ is Fermi velocity. Below we assume $Z \gg 1$. The schematic illustration of the models is shown in Fig. 1. Here, $\alpha$ and $\beta$ denote the angles between the normal to the interface and the crystal axis of the left and right $d$-wave (or $p$-wave) superconduct- ors, respectively. The lobe direction of the pair potential and the direction of the crystal axis are chosen to be the same. The pair potential along the quasiparticle trajectory with the injection angle $\phi$ is given by $\Delta_{L}=\Delta \cos [2(\phi-\alpha)] \exp (-i \Psi)$ and $\Delta_{R}=\Delta \cos [2(\phi-\beta)]$ for the left and the right superconductor, respectively. For ballistic junctions, we use a similar model without DN and calculate the Josephson current following Ref. 9.

We parametrize the quasiclassical Green functions $G$ and $F$ with a function $\Phi_{\omega} \cdot{ }^{23,24}$

$$
G_{\omega}=\frac{\omega}{\sqrt{\omega^{2}+\Phi_{\omega} \Phi_{-\omega}^{*}}}, \quad F_{\omega}=\frac{\Phi_{\omega}}{\sqrt{\omega^{2}+\Phi_{\omega} \Phi_{-\omega}^{*}}},
$$

where $\omega$ is the Matsubara frequency. Then the Usadel equation reads $^{25}$

$$
\xi^{2} \frac{\pi T_{C}}{\omega G_{\omega}} \frac{\partial}{\partial x}\left(G_{\omega}^{2} \frac{\partial}{\partial x} \Phi_{\omega}\right)-\Phi_{\omega}=0
$$

with the coherence length $\xi=\sqrt{D / 2 \pi T_{C}}$, the diffusion constant $D$ and the transition temperature $T_{C}$. We solve the Usadel equation with the boundary conditions in Ref. 20 at $x$ $= \pm L$ and those in Ref. 26 at $x=0$.

The Josephson current is given by

$$
\frac{e I R}{\pi T_{C}}=i \frac{R T L}{2 R_{d} T_{C}} \sum_{\omega} \frac{G_{\omega}^{2}}{\omega^{2}}\left(\Phi_{\omega} \frac{\partial}{\partial x} \Phi_{-\omega}^{*}-\Phi_{-\omega}^{*} \frac{\partial}{\partial x} \Phi_{\omega}\right),
$$

where $T$ is temperature and $R \equiv 2 R_{d}+R_{b}+2 R_{b}^{\prime}$ is the normal state resistance of the junction. In the following we focus on the $I_{C} R$ value where $I_{C}$ denotes the magnitude of the maximum Josephson current. We fix parameters as $Z^{\prime}=0, R_{d} / R_{b}$ $=0.01, R_{d} / R_{b}^{\prime}=10$, and $E_{T h} / \Delta_{0}=1$ for $D / \mathrm{DN} / I / \mathrm{DN} / D$ junctions and $Z=10$ for $D I D$ junctions. $\Delta_{0}$ denotes the value of $\Delta$ at zero temperature. The choice of the small magnitude of $Z^{\prime}$ and $R_{b}^{\prime}$ and large Thouless energy is justified by the fact that thin DN could be naturally formed due to the degradation of superconductivity near the interface.

The $\alpha$ dependence of $I_{C} R$ for $d$-wave superconductor junctions is plotted in Fig. 2. In Figs. 2(a) and 2(b), $I_{C} R$ of ballistic junctions is plotted for low $\left(T / T_{C}=0.2\right)$ and high temperature $\left(T / T_{C}=0.9\right)$, respectively. With the increase of the magnitude of $\beta$, the dependence of $I_{C} R$ on $\alpha$ transforms from $\cos 2 \alpha$ to $\sin 2 \alpha$. These $\alpha$ dependences cannot be expressed by the SR formula, where $I_{C} R$ is proportional to $\cos 2 \alpha$ for fixed $\beta$. On the other hand in $D / \mathrm{DN} / I / \mathrm{DN} / D$ junctions, $I_{C} R$ has a simple form, $\cos 2 \alpha$, independent of $\beta$ at low and high temperatures as shown in Figs. 2(c) and 2(d), respectively. The magnitudes of $I_{C} R$ in $D / \mathrm{DN} / I / \mathrm{DN} / D$ junctions are at least two orders smaller than those in $D I D$ junctions. By taking account of the $\beta$ dependence, $I_{C} R$ is almost proportional to $\cos 2 \alpha \cos 2 \beta$. It should be remarked that this fitting is possible for small magnitude of $Z^{\prime}$ and $R_{b}^{\prime} / R_{d}$ where the MARS formed at the $D / \mathrm{DN}$ interface do not influence seriously the charge transport.

The corresponding results of $I_{C} R$ for $p$-wave superconductor junctions are plotted in Fig. 3. For $P / \mathrm{DN} / I / \mathrm{DN} / P$ junctions, $I_{C} R$ can be fitted by $\cos \alpha \cos \beta$. Similar to the case of $d$-wave junctions, this fitting is possible for small 

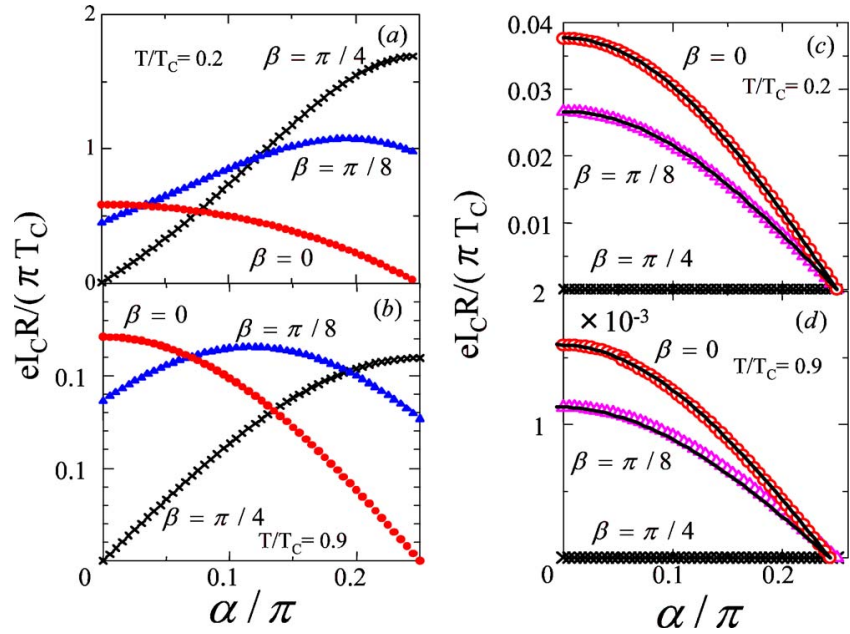

FIG. 2. (Color online) Maximum Josephson current for $d$-wave junctions. (a) and (b) $D I D$ junctions. (c) and (d) $D / D N / I / D N / D$ junctions. Solid lines in (c), (d) are proportional to $\cos 2 \alpha \cos 2 \beta$.

magnitude of $Z^{\prime}$ and $R_{b}^{\prime} / R_{d}$. For ballistic junctions, as shown in Figs. 3(a) and 3(b), this fitting does not work any more.

In the following, we will present analytical result demonstrating why the SR formula does not work in ballistic junctions and works in the diffusive junctions. Although we focus on $d$-wave junctions, similar discussion is possible for $p$-wave junctions. Near $T_{C}(\Delta \ll \omega)$, we can get the formula for the ballistic DID junctions ${ }^{9}$

$$
\begin{gathered}
\frac{e I R}{\pi T_{C}}=\frac{\Delta^{2} \sin \Psi}{8 T T_{C}} F_{0}, \\
F_{0}=\left\langle\cos ^{2} 2 \phi\right\rangle \cos 2 \alpha \cos 2 \beta+\left\langle\sin ^{2} 2 \phi\right\rangle \sin 2 \alpha \sin 2 \beta .
\end{gathered}
$$

Here, the average over the various angles of injected particles at the interfaces is defined as
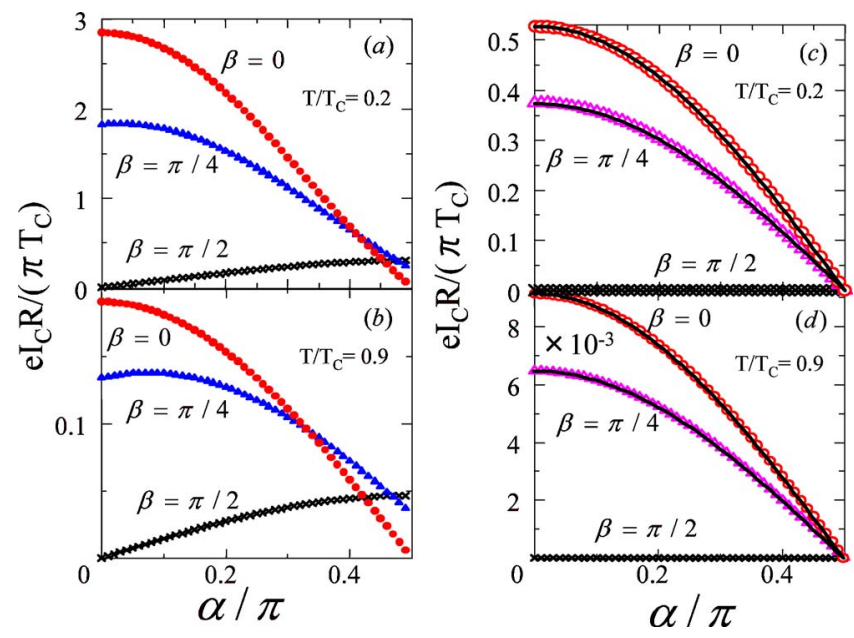

FIG. 3. (Color online) Maximum Josephson current for $p$-wave junctions. (a) and (b) PIP junctions. (c) and (d) $P / D N / I / D N / P$ junctions with solid lines that are proportional to $\cos \alpha \cos \beta$.

$$
\langle B(\phi)\rangle=\frac{\int_{-\pi / 2}^{\pi / 2} d \phi T(\phi) \cos \phi B(\phi)}{\int_{-\pi / 2}^{\pi / 2} d \phi T(\phi) \cos \phi}
$$

with $T(\phi)=T_{m}$. It is easy to check that $\left\langle\cos ^{2} 2 \phi\right\rangle$ and $\left\langle\sin ^{2} 2 \phi\right\rangle$ are of the same order for all $Z$. Therefore, SR formula cannot be applicable to nonzero values of $\alpha$ and $\beta$. Also we can roughly estimate the Josephson current

$$
\frac{e I R}{\pi T_{C}} \cong \frac{\Delta^{2}}{16 T T_{C}} \cos (2 \alpha-2 \beta) \sin \Psi
$$

which is consistent with the result in Fig. 2(b). In the case of $P I P$ junctions, we can obtain the corresponding equation by replacing $2 \alpha, 2 \beta$, and $2 \phi$ with $\alpha, \beta$, and $\phi$ in the above equations.

Next we consider the $D / \mathrm{DN} / I / \mathrm{DN} / D$ junctions. Near $T_{C}$, we can linearize the Usadel equation as follows:

$$
\xi^{2} \frac{\partial^{2}}{\partial x^{2}} \Phi_{j \omega}-\frac{\omega}{\pi T_{C}} \Phi_{j \omega}=0
$$

where $j(=1,2)$ denotes the left or right DN. Similarly the boundary conditions at $x=-L, x=0$, and $x=L$ are reduced to

$$
\begin{gathered}
\left.\frac{\partial}{\partial x} \Phi_{1 \omega}\right|_{x=-L}=-\left.\frac{R_{d}}{R_{b}^{\prime} L}\left(-\Phi_{1 \omega}+I_{0} \cos 2 \alpha e^{-i \Psi}\right)\right|_{x=-L}, \\
\left.\frac{\partial \Phi_{1 \omega}}{\partial x}\right|_{x=0}=\left.\frac{\partial \Phi_{2 \omega}}{\partial x}\right|_{x=0}=\left.\frac{R_{d}\left(\Phi_{2 \omega}-\Phi_{1 \omega}\right)}{R_{b} L}\right|_{x=0}, \\
\left.\frac{\partial \Phi_{2 \omega}}{\partial x}\right|_{x=L}=\left.\frac{R_{d}}{R_{b}^{\prime} L}\left(-\Phi_{2 \omega}+I_{0} \cos 2 \beta\right)\right|_{x=L}
\end{gathered}
$$

with $I_{0}=\Delta\langle\cos 2 \phi\rangle$.

Solving the above equations, we find the expression for the Josephson current of the form

$$
\begin{gathered}
\frac{e I R}{\pi T_{C}}=\frac{R r r^{\prime 2}}{R_{d}} \frac{T}{T_{C}} \sum_{\omega} \frac{\gamma L\langle\cos 2 \phi\rangle^{2} \Delta^{2} \cos 2 \alpha \cos 2 \beta \sin \Psi}{\omega^{2} F_{1} F_{2}}, \\
F_{1}=\gamma L \sinh \gamma L+r^{\prime} \cosh \gamma L, \\
F_{2}=\left[\left(2 r r^{\prime}+\gamma^{2} L^{2}\right) \sinh \gamma L+\left(2 r+r^{\prime}\right) \gamma L \cosh \gamma L\right]
\end{gathered}
$$

with $r=\frac{R_{d}}{R_{b}}, r^{\prime}=\frac{R_{d}}{R_{b}^{\prime}}$, and $\gamma=\sqrt{\frac{2 \omega}{D}}$. Thus the SR formula is proven to be valid near $T_{C}$. In the case of $P / \mathrm{DN} / I / \mathrm{DN} / P$ junctions, $\cos 2 \phi, \cos 2 \alpha$, and $\cos 2 \beta$ have to be replaced with $\cos \phi, \cos \alpha$, and $\cos \beta$, respectively, to obtain the corresponding formula. This result is consistent with the previous study of DID junctions with rough interface, ${ }^{27}$ where the SR formula is applicable as well.

In order to understand the above results qualitatively, let us discuss the symmetry of the pair potential by the inversion operation around the plane perpendicular to the interface. As shown in Fig. 4, $d_{x^{2}-y^{2}}$ wave and $p_{x}$ wave are symmetric while $d_{x y}$ wave and $p_{y}$ wave are antisymmetric by this operation. Only the symmetric pair wave function is taken into 


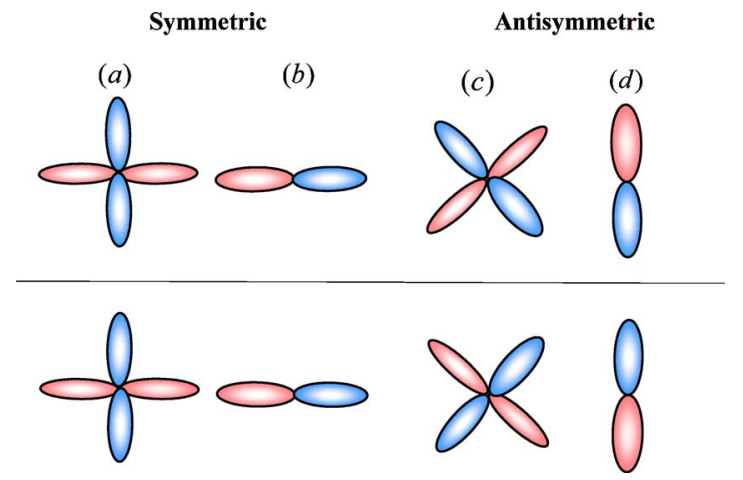

FIG. 4. (Color online) Schematic illustration of the inversion symmetry of the pair potential around the plane normal to the interface. (a) $d_{x^{2}-y^{2}}$ wave, (b) $p_{x}$ wave, (c) $d_{x y}$ wave, and (d) $p_{y}$ wave. The $\phi$ dependences are given by $\cos 2 \phi, \cos \phi, \sin 2 \phi$, and $\sin \phi$.

account in the SR formula. Applying this idea to an arbitrary unconventional superconductor with angular momentum $l$, one can argue that the Josephson current is proportional to $\cos l \alpha \cos l \beta$. It is straightforward to get this result just by replacing $\cos 2 \phi, \cos 2 \alpha$, and $\cos 2 \beta$ with $\cos l \phi, \cos l \alpha$, and $\cos l \beta$ in Eq. (11), respectively.

In summary, we have studied the validity and the physical meaning of the Sigrist-Rice formula in $d$-wave superconductor junctions. According to the SR formula, the amplitude of the maximum Josephson current is proportional to $\cos 2 \alpha \cos 2 \beta$. Although this formula is not applicable to the ballistic junctions, it works well for $D / \mathrm{DN} / I / \mathrm{DN} / D$ junctions where the DN regions are located between the $d$-wave superconductor and the insulator. We have also shown that in $P / \mathrm{DN} / I / \mathrm{DN} / P$ junctions, the Josephson current is proportional to $\cos \alpha \cos \beta$. The obtained results may help to obtain information about pairing symmetry in experiments with unconventional superconducting junctions.

T. Y. acknowledges support by the JSPS. This work is supported by Grant-in-Aid for Scientific Research on Priority Area "Novel Quantum Phenomena Specific to Anisotropic Superconductivity" (Grant No. 17071007) from the Ministry of Education, Culture, Sports, Science and Technology of Japan.
${ }^{1}$ C. C. Tsuei and J. R. Kirtley, Rev. Mod. Phys. 72, 969 (2001).

${ }^{2}$ D. J. Van Harlingen, Rev. Mod. Phys. 67, 515 (1995).

${ }^{3}$ M. Sigrist and T. M. Rice, Rev. Mod. Phys. 67, 503 (1995).

${ }^{4}$ S. Kashiwaya and Y. Tanaka, Rep. Prog. Phys. 63, 1641 (2000).

${ }^{5}$ M. Sigrist and T. M. Rice, J. Phys. Soc. Jpn. 61, 4283 (1992).

${ }^{6}$ L. J. Buchholtz and G. Zwicknagl, Phys. Rev. B 23, 5788 (1981); J. Hara and K. Nagai, Prog. Theor. Phys. 74, 1237 (1986); C. Bruder, Phys. Rev. B 41, 4017 (1990); C. R. Hu, Phys. Rev. Lett. 72, 1526 (1994).

${ }^{7}$ Y. Tanaka and S. Kashiwaya, Phys. Rev. Lett. 74, 3451 (1995).

${ }^{8}$ Y. Tanaka and S. Kashiwaya, Phys. Rev. B 56, 892 (1997).

${ }^{9}$ Y. Tanaka and S. Kashiwaya, Phys. Rev. B 53, R11957 (1996).

${ }^{10}$ Yu. S. Barash, H. Burkhardt, and D. Rainer, Phys. Rev. Lett. 77, 4070 (1996).

${ }^{11}$ G. Testa, E. Sarnelli, A. Monaco, E. Esposito, M. Ejrnaes, D.-J. Kang, S. H. Mennema, E. J. Tarte, and M. G. Blamire, Phys. Rev. B 71, 134520 (2005).

${ }^{12}$ E. Ilichev, M. Grajcar, R. Hlubina, R. P. J. I. Jsselsteijn, H. E. Hoenig, H.-G. Meyer, A. Golubov, M. H. S. Amin, A. M. Zagoskin, A. N. Omelyanchouk, and M. Yu. Kuprianov, Phys. Rev. Lett. 86, 5369 (2001).

${ }^{13}$ F. Lombardi, F. Tafuri, F. Ricci, F. Miletto Granozio, A. Barone, G. Testa, E. Sarnelli, J. R. Kirtley, and C. C. Tsuei, Phys. Rev. Lett. 89, 207001 (2002).

${ }^{14}$ T. Bauch, F. Lombardi, F. Tafuri, A. Barone, G. Rotoli, P. Delsing, and T. Claeson, Phys. Rev. Lett. 94, 087003 (2005).
${ }^{15}$ Soon-Gul Lee and Yunseok Hwang, Appl. Phys. Lett. 76, 2755 (2000).

${ }^{16}$ W. K. Neils et al., Physica C 368, 261 (2002).

${ }^{17}$ H. J. H. Smilde, A. A. Golubov, Ariando, G. Rijnders, J. M. Dekkers, S. Harkema, D. H. A. Blank, H. Rogalla, and H. Hilgenkamp, Phys. Rev. Lett. 95, 257001 (2005).

${ }^{18}$ J. R. Kirtley et al., Nat. Phys. 2, 190 (2006).

${ }^{19}$ Y. Tanaka, Y. V. Nazarov, and S. Kashiwaya, Phys. Rev. Lett. 90, 167003 (2003); Y. Tanaka, Yu. V. Nazarov, A. A. Golubov, and S. Kashiwaya, Phys. Rev. B 69, 144519 (2004).

${ }^{20}$ T. Yokoyama, Y. Tanaka, A. A. Golubov, and Y. Asano, Phys. Rev. B 73, 140504(R) (2006).

${ }^{21}$ Y. Tanaka and S. Kashiwaya, Phys. Rev. B 70, 012507 (2004); Y. Tanaka, S. Kashiwaya, and T. Yokoyama, ibid. 71, 094513 (2005); Y. Tanaka, Y. Asano, A. A. Golubov, and S. Kashiwaya, ibid. 72, 140503(R) (2005).

${ }^{22}$ Y. Asano, Y. Tanaka, and S. Kashiwaya, Phys. Rev. Lett. 96, 097007 (2006); Y. Asano, Y. Tanaka, T. Yokoyama, and S. Kashiwaya, Phys. Rev. B 74, 064507 (2006).

${ }^{23}$ K. K. Likharev, Rev. Mod. Phys. 51, 101 (1979).

${ }^{24}$ A. A. Golubov, M. Yu. Kupriyanov, and E. Il'ichev, Rev. Mod. Phys. 76, 411 (2004).

${ }^{25}$ K. D. Usadel, Phys. Rev. Lett. 25, 507 (1970).

${ }^{26}$ M. Yu. Kupriyanov and V. F. Lukichev, Zh. Eksp. Teor. Fiz. 94, 139 (1988) [Sov. Phys. JETP 67, 1163 (1988)].

${ }^{27}$ A. A. Golubov and M. Yu. Kupriyanov, JETP Lett. 67, 501 (1998); 69, 262 (1999). 